\title{
Improving the safety of platelet transfusions by UV-C: let's go back to the bench
}

\author{
Daniele Prati \\ Department of Transfusion Medicine and Hematology, Fondazione IRCCS Ca' Granda Ospedale Maggiore Policlinico, Milano, Italy \\ E-mail: DANIELE PRATI - daniele.prati@policlinico.mi.it
}

doi:10.3324/haematol.2020.275156

T echnologies for pathogen reduction in blood components have been under development for more than 30 years, with the aim of mitigating the infectious risks of blood transfusion. They are based on the principle of inactivating all nucleic acids in the blood unit (including intra-cellular), to prevent the replication of any possible pathogen. This is particularly desirable to protect platelet transfusion recipients, who are at higher risk of septic reactions. In fact platelets - differently from other blood products which are kept refrigerated or frozen - are stored at room temperature, thus increasing the risk of bacterial growth.

As recently outlined, designing and conducting clinical trials on pathogen reduced platelets is not straightforward. ${ }^{1,2}$ In principle, studies should aim to demonstrate that pathogenreduced platelets are more effective than standard platelets in preventing transfusion-transmitted infections. However, demonstrating such an advantage is considered unrealistic: given the unprecedented levels of blood safety, too many participants would need to be enrolled to achieve an adequate statistical power. Thus, the antimicrobial efficacy of these techniques is taken for granted from in vitro studies, and aims are set on the efficacy of platelet transfusion: i.e., whether or not pathogen-reduced products retain their ability to increase platelet count, prevent bleeding and do not overly increase product need. Trials are generally based on a non-inferiority hypothesis, because pathogen-reduced platelets are not expected to provide better hemostatic efficacy than conventional platelets. ${ }^{3}$

Two pathogen-reduction techniques based on photochemical treatment of platelets, amotosalen plus UV-A light (Intercept, Cerus) and riboflavin plus UV light (Mirasol, TerumoBCT) - have already been tested in several randomized studies of prophylactic transfusion in thrombocytopenic patients. As summarized in a Cochrane systematic review the treatment with either of these two methods does not seem to cause higher rates of bleeding, death, or serious adverse events in recipients. ${ }^{2}$ However, it is associated with approximately $20 \%$ lower post transfusion platelet count increments, shorter transfusion intervals and higher rates of refractoriness to platelet transfusions. ${ }^{2,4}$ This, together with concerns about the long-term safety profile of amotosalen or riboflavin and cost, have hampered the widespread introduction of pathogen-reduction techniques in many countries.

Another pathogen reduction method, the Theraflex system (Macopharma S.A.S.), has more recently been developed. In contrast with Mirasol and Intercept, it is based on simple UV-C irradiation of platelets, without the addition of photoactive substances. The article by Brixner and colleagues in the current issue of Haematologica ${ }^{5}$ reports the results of the first clinical study comparing the efficacy and safety of UV-C treated platelets to standard platelets (the CAPTURE trial). In a non-inferiority trial, the working group selected as primary endpoint the 1-hour corrected count increment (CCI), a measure of response to platelet transfusion that "corrects" the post-transfusion increase of platelet count for blood volume and number of platelets transfused, and set the acceptable inferiority margin at $30 \%$.

From a methodological point of view, the trial was well designed and well conducted, and the authors should be commended for their effort. The main sponsor of the study was a non-commercial institution, the Research Foundation of the German Red Cross Blood Services. The working group successfully enrolled 175 patients (slightly more than the 166 planned), in 10 clinical centers. Patients were evaluated in up to eight per-protocol platelet transfusion episodes, and the percentage of off-protocol transfusions was kept low (about $5 \%$ in both arms). Both aphaeresis and buffy-coat derived platelet pools were used, reflecting the standard transfusion practice in Europe. Perhaps, the main limitation of the CAPTURE study was the choice of 1-hour CCI as primary endpoint. CCI is commonly used as a surrogate outcome for platelet transfusion efficacy, but its correlation with clinical efficacy has not been documented. ${ }^{1}$ Theoretically, bleeding endpoints graded according to World Health Organisation system would have been more appropriate. However, reliable grading is not easy to standardise and apply, especially in a context of independent studies involving multiple evaluation sites. ${ }^{1,3,6}$ However, CCI has been used in most previous trials on platelet concentrate pathogenreduction, ${ }^{2}$ which makes it acceptable for this initial evaluation of the Theraflex system.

The results of the CAPTURE trial are of great interest. In an intent-to-treat analysis, the mean 1-hour CCI was 12.7 (95\% CI: 11.42-13.97) in the patients receiving UV-C treated products, and 15.53 (95\% CI: 14.88-16.88) in those receiving conventional platelets. This accounted for a mean difference of $18.24 \%$ (95\% CI: 6.4-30.8) between the two groups. Similar results were obtained using a per-protocol analysis. Thus, non-inferiority of pathogen-reduced platelets compared to the standard of care cannot be claimed, despite a narrow margin well below the pre-trial defined limit of $30 \%$. In other words UV-C-treated platelets were clearly less effective than standard platelets in increasing post transfusion counts. In addition, patients in the experimental treatment arm received $25 \%$ more platelet transfusions, seriously affecting treatment costs, and patients receiving pathogentreated platelets had a higher frequency of low-grade transfusion-related adverse events (probably related to the higher transfusion requirements). No differences between the two treatment arms were observed with regards to the incidence of platelet alloimmunization and serious adverse events (including severe bleeding episodes), but it should be emphasized that the trial was not adequately powered for detecting them. Therefore, as correctly stated by the authors, no firm conclusions on safety could be drawn on the basis of the CAPTURE data.

Theraflex received the CE mark in 2009, but has not yet been commercialized. Certainly the evidence emerging from 
the CAPTURE study mitigates the introduction of this new technology in clinical practice. Well designed clinical trials obtain their credibility from the definition of a priori hypotheses that helps researchers to avoid drawing wrong conclusions, and negative results are as useful as positive results in guiding medical treatments.

However, even when the primary outcome of a clinical trial fails, new research opportunities open up. ${ }^{8}$ Hopefully, a careful analysis of the CAPTURE data will lead to future research in the field. Additional laboratory studies are probably required to i) gain further insight into the damage that UV-C irradiation causes to platelets apart from pathogen inactivation and to ii) develop strategies to improve the quality of Theraflex treated products.

Concerns regarding the possible transfusion transmission of SARS-CoV-2 at the beginning of the ongoing pandemic have revamped the interest in approaches capable of protecting the blood supply from known and newly emerging threats. ${ }^{9}$ As Brixen and colleagues remind us, safe and effective pathogen reduction methods are still an unmet need.

\section{Disclosures}

$D P$ sits on advisory boards, has received travel or research grants, as well as speaking and teaching fees from Macopharma,
Ortho Clinical Diagnostics, Grifols, Terumo, Immucor, Diamed, Diatech Pharmacogenetics.

\section{References}

1. Heddle NM, Cardoso M, van der Meer PF. Revisiting study design and methodology for pathogen reduced platelet transfusions: a round table discussion. Transfusion. 2020;60(7):1604-1611.

2. Rebulla P, Garban F, van der Meer PF, Heddle NM, McCullough J. A crosswalk tabular review on methods and outcomes from randomized clinical trials using pathogen reduced platelets. Transfusion. 2020;60(6):1267-1277.

3. Rebulla $\mathrm{P}$. The long and winding road to pathogen reduction of platelets, red blood cells and whole blood. Br J Haematol. 2019;186(5):655-667

4. Estcourt LJ, Malouf R, Hopewell S, et al. Pathogen-reduced platelets for the prevention of bleeding. Cochrane Database Syst Rev. 2017;7(7):CD009072

5. Brixner V, Bug G, Pohler P, et al. Efficacy of UVC-treated, pathogenreduced platelets versus untreated platelets: a randomized controlled non-inferiority trial. Haematologica. 2021;106(4):1086-1096.

6. Rebulla P, Vaglio S, Beccaria F, et al. Clinical effectiveness of platelets in additive solution treated with two commercial pathogen-reduction technologies. Transfusion. 2017;57(5):1171-1183.

7. Mauri L, D'Agostino RB Sr. Challenges in the design and interpretation of noninferiority trials. N Engl J Med. 2017;377(14):1357-1367.

8. Pocock SI, Stone GW. The primary outcome fails - what next? N Engl J Med. 2016;375(9):861-870.

9. Stanworth SJ, New HV, Apelseth TO, et al. Effects of the COVID-19 pandemic on supply and use of blood for transfusion. Lancet Haematol. 2020;7(10):e756-e764.

\title{
Understanding how retinoic acid derivatives induce differentiation in non-M3 acute myelogeneous leukemia
}

\author{
Martin Carroll \\ Division of Hematology and Oncology, University of Pennsylvania, Philadelphia, PA, USA \\ E-mail: MARTIN CARROLL - carroll2@pennmedicine.upenn.edu
}

doi:10.3324/haematol.2020.275412

$\mathrm{O}$ ver 30 years ago, Huang and colleagues published the startling result that all trans-retinoic acid (ATRA) could induce clinical remissions without myelosuppression in patients with acute promyelocytic leukemia (APML). ${ }^{1}$ Analysis in this report and subsequent analysis demonstrated that responses are due to induced differentiation of the leukemic clone and not the induction of cell death in the malignant cells. This work introduced the concept of differentiation therapy to the world of leukemia therapeutics. Other recently developed therapeutics for acute myeloid leukemia (AML) including FLT3 and IDH inhibitors in some patients with the targeted mutations are now known to induce differentiation. ${ }^{2,3}$ However, there remain two outstanding questions in this field that stem from those original remarkable observations. First, what is the role of retinoic acid or its derivatives in controlling normal myeloid maturation? Second, how can this information be used to develop retinoic acid based therapeutics for non-M3 AML? di Martino and colleagues provide exciting new insights into these questions in this issue of Haematologica. ${ }^{4}$

To understand the complexity of these questions, it is valuable to first briefly introduce how retinoids and their derivatives function. Conceptually, retinoic acid (RA) functions through one of the retinoic acid receptors (RAR) which are members of the nuclear hormone receptor family. To simplify, binding of RA to the RAR induces binding to DNA. Commonly, this binding leads to recruitment of factors that promote gene transcription (such as histone acetyl transferases) and displacement of inhibitors of transcription such as nuclear receptor corepressor (NCOR1). There are many levels of complexity in these gene regulatory events. ${ }^{5}$ Importantly, there are actually three isoforms of RAR. RAR can function as homodimers, heterodimers with themselves or heterodimers with other members of the nuclear hormone receptor superfamily including retinoic X receptors (RXR), Vitamin $\mathrm{D}$ receptors (VDR) and peroxisome proliferator activated receptor (PPAR). Thus, there are many combinatorial possibilities for gene targets and multiple levels of redundancy that have made defining the specific role of the nuclear hormone receptor superfamily in myeloid maturation and leukemia therapy challenging.

To address these questions, Di Martino and colleagues first use a murine model of AML induced using the KMT2A fusion protein, KMT2A-AF9. The leukemic cells were transduced with reporter constructs that are quite specific for activation by isoforms of RAR or RXR, trans- 